\title{
Quantum Treatment of the Multiple Scattering and Collective Flow in Intensity Interferometry
}

\author{
Cheuk-Yin Wong \\ Physics Division, Oak Ridge National Laboratory, Oak Ridge, TN 37830 \\ Physics Department, University of Tennessee, Knoxville, TN 37996
}

\begin{abstract}
We apply the path-integral method to study the multiple scattering and collective flow in intensity interferometry in high-energy heavy-ion collisions. We show that the Glauber model and eikonal approximation in an earlier quantum treatment are special examples of the more general path-integral method. The multiple scattering and collective flow lead essentially to an initial source at a shifted momentum, with a multiple collision absorption factor that depends on the pion absorption cross section and a phase factor that depends on the deviations of the in-medium particle momenta from their asymptotic values.
\end{abstract}

\section{INTRODUCTION}

The intensity interferometry, as first proposed by Hanbury-Brown-Twiss (HBT) to measure the angular diameter of a star [1], has been applied to optical coherence [2], subatomic physics, and nuclear collisions [3]. In HBT measurements in high-energy heavyion collisions, the initial source particles undergo multiple scattering and collective flow. As a result, it is conventionally assumed that the initial chaotic source evolves into a different chaotic source distribution at thermal freeze-out which becomes the distribution measured in intensity interferometry. This conventional assumption is recently subject to question. Because the intensity interferometry is purely a quantum-mechanical phenomenon, the multiple scattering and collective flow must be investigated within a quantum-mechanical framework $[4,5,6]$. Applying the Glauber multiple scattering theory at high energies and the optical model at lower energies, we find that multiple scatterings lead essentially to an initial source distribution with absorption $[4,5,6]$. Using the Feynman path integral method, we further find that the collective flow leads to a phase factor that depends on the deviations of the in-medium particle momenta from their asymptotic values. Subsequent work by Kapusta and Li [7] supports qualitatively some of the earlier results of Ref. [4]. Following this suggestion of a quantum treatment, Cramer and his collaborators [8] later considered the effects of produced pions in a static optical model potential. The work of Cramer et al. [8] is however incomplete, as the effects of particle collisions and the collective flow have not been taken into account.

In this manuscript, we apply the path-integral method for the quantum treatment of the multiple scattering and collective flow in intensity interferometry. We show that the Glauber model and eikonal approximation in an earlier quantum treatment of the multiple scattering $[4,5]$ are special examples of the more general path-integral method. 


\section{PION ENVIRONMENT AND ELASTIC SCATTERING}

Before we present a quantum treatment of intensity interferometry, it is illuminating to investigate the pion environment after a chaotic pion source is produced in the phase transition of a quark-gluon plasma.

At the phase transition temperature of $T \sim 180 \mathrm{MeV}$, the average pion density is $\sim 0.3$ pions/fm ${ }^{3}$, the pion energy is $\sim 0.5 \mathrm{GeV}$, and the average C.M. energy, $\left\langle\sqrt{s_{\pi \pi}}\right\rangle$, in a $\pi-\pi$ collision is $\sim 0.7 \mathrm{GeV}$. Thus the pion source is dense and pions are energetic after their production. As $\left\langle\sqrt{s_{\pi}}\right\rangle$ is only slightly lower than the $\rho$ mass, a substantial fraction of $\pi$ - $\pi$ collisions will go through the $I=1, \rho$ resonance. The width of the $\rho$ resonance is $150 \mathrm{MeV}$ in free space. In a thermalized medium at $T=150 \mathrm{MeV}$, the width increases substantially to $\sim 300 \mathrm{MeV}$ [9], and the $\rho$ resonance mean lifetime in the medium becomes $\sim \hbar /(300 \mathrm{MeV})$ or $0.67 \mathrm{fm} / \mathrm{c}$. The orbiting time for the $\rho$ meson is of order $2 \pi r$ and $2 r \sim 0.77 \mathrm{fm} \mathrm{[10].} \mathrm{As} \mathrm{the} \rho$ meson mean lifetime in the medium $(\sim 0.67 \mathrm{fm} / \mathrm{c})$ is much shorter than its orbiting time $(\sim 2.4 \mathrm{fm} / \mathrm{c})$, it is unlikely for an $I=1, \pi-\pi$ pair to complete an orbital revolution before the $\rho$ meson breaks apart into two pions. The scattering of the two pions through the intermediary $\rho$ meson is essentially an elastic scattering with an energy-dependent amplitude. Furthermore, the $\pi \pi \rightarrow K \bar{K}$ threshold is $\sim 1 \mathrm{GeV}$, substantially greater than $\left\langle\sqrt{s_{\pi \pi}}\right\rangle$. Hence, chemical reactions of pions in the medium are essentially completed very shortly after the phase transition. From the state of chemical freeze-out to thermal freeze-out, the scatterings suffered by the pions are predominately elastic.

\section{PATH INTEGRAL METHOD FOR INTENSITY INTERFEROMETRY}

As a pion propagates from the state of chemical freeze-out to thermal freeze-out, the elastic $\pi \pi$ scattering can be described by short-ranged scalar and vector interactions,

$v_{\mathrm{col}}^{(s, v)}\left(q-q_{i}\right)$, where $q$ is the coordinate of the propagating pion and $q_{i}$ is the coordinate of a pion in the medium. The propagating pion is also subject to a collective flow which can be described by a long-range density-dependent mean-field scalar and vector interactions, $V_{\mathrm{mf}}^{(s, v)}(q)$, as in similar cases in the dynamics of the nuclear fluid [11]. The Lagrangian for the propagating pion is given by

$$
L(q, \dot{q})=L_{\mathrm{mf}}(q, \dot{q})+L_{\mathrm{col}}(q, \dot{q}),
$$

where

$$
\begin{gathered}
L_{\mathrm{mf}}(q, \dot{q})=-\left[m_{\pi}+V_{\mathrm{mf}}^{(s)}(q)\right] \sqrt{1-\dot{\mathbf{q}}^{2}}+\dot{\mathbf{q}} \cdot \mathbf{V}_{\mathrm{mf}}^{(v)}(q)-V_{\mathrm{mf}}^{0(v)}(q), \\
L_{\mathrm{col}}(q, \dot{q})=-V_{\mathrm{col}}^{(s)}(q) \sqrt{1-\dot{\mathbf{q}}^{2}}+\dot{\mathbf{q}} \cdot \mathbf{V}_{\mathrm{col}}^{(v)}(q)-V_{\mathrm{col}}^{0(v)}(q), \\
V_{\mathrm{col}}^{(s, v)}(q)=\sum_{i}^{N_{\pi}-1} v_{\mathrm{col}}^{(s, v)}\left(q-q_{i}\right) .
\end{gathered}
$$

From the Lagrangian of the propagating pion, one obtains the pion three-momentum,

$$
\mathbf{p}=\partial L / \partial \dot{\mathbf{q}}=\gamma\left[m_{\pi}+V^{(s)}(q)\right] \dot{\mathbf{q}}+\mathbf{V}^{(v)}(q),
$$


the pion Hamiltonian,

$$
H=p^{0}=\mathbf{p} \cdot \dot{\mathbf{q}}-L=\gamma\left[m_{\pi}+V^{(s)}(q)\right]+V^{0(v)}(q),
$$

and the pion mass-shell condition,

$$
\left[p^{0}-V^{0(v)}(q)\right]^{2}-\left[\mathbf{p}^{2}-\mathbf{V}^{(v)}(q)\right]^{2}-\left[m_{\pi}+V^{(s)}(q)\right]^{2}=0,
$$

where $\gamma=1 / \sqrt{1-\dot{\mathbf{q}}^{2}}$ and $V^{(s, v)}(q)=V_{\mathrm{mf}}^{(s, v)}(q)+V_{\text {col }}^{(s, v)}(q)$. For a pion produced at $x$ with momentum $\kappa$ to propagate in the pion medium to the thermal freeze-out point $x_{f}$ and be detected at the detecting point $x_{d}$ with momentum $k$, the probability amplitude is [12]

$$
K\left(\kappa x \rightarrow k x_{d}\right)=\int \mathscr{D} q e^{i S\left(\kappa x \rightarrow k x_{d} ; q\right)},
$$

where $\int \mathscr{D} q \ldots$ is the sum over all paths $q$ from $x$ to $x_{d}$, and the action $S\left(\kappa x \rightarrow k x_{d} ; q\right)$ is

$$
S\left(\kappa x \rightarrow k x_{d} ; q\right)=\int L(q, \dot{\mathbf{q}}) d t=-\int_{x}^{x_{d},(\text { ath } q)} p\left(q^{\prime}\right) \cdot d q^{\prime} .
$$

We can separate $S\left(\kappa x \rightarrow k x_{d} ; q\right)$ into different contributions,

$$
\begin{gathered}
S\left(\kappa x \rightarrow k x_{d} ; q\right)=-k \cdot\left(x_{d}-x\right)+\delta_{\mathrm{mf}}\left(\kappa x, k x_{d} ; q\right)+\delta_{\mathrm{col}}\left(\kappa x, k x_{d} ; q\right) \\
\delta_{\mathrm{mf}}\left(\kappa x, k x_{d} ; q\right)=-\int_{x}^{x_{d},(\operatorname{path} q)}\left[p_{\mathrm{mf}}\left(q^{\prime}\right)-k\right] \cdot d q^{\prime}=-\int_{x}^{x_{f},(\operatorname{path} q)}\left[p_{\mathrm{mf}}\left(q^{\prime}\right)-k\right] \cdot d q^{\prime}, \\
\delta_{\mathrm{col}}\left(\kappa x, k x_{d} ; q\right)=-\int_{x}^{x_{d},(\operatorname{path} q)} p_{\mathrm{col}}\left(q^{\prime}\right) \cdot d q^{\prime}=-\int_{x}^{x_{f},(\operatorname{path} q)} p_{\mathrm{col}}\left(q^{\prime}\right) \cdot d q^{\prime} \\
p_{\mathrm{mf}}(q)=\left(\gamma\left[m_{\pi}+V_{\mathrm{mf}}^{(s)}(q)\right]+V_{\mathrm{mf}}^{0(v)}(q), \gamma\left[m_{\pi}+V_{\mathrm{mf}}(q)\right] \dot{\mathbf{q}}+\mathbf{V}_{\mathrm{mf}}^{(v)}(q)\right) \\
p_{\mathrm{col}}(q)=\left(\gamma V_{\mathrm{col}}^{(s)}(q)+V_{\mathrm{col}}^{0(v)}(q), \gamma V_{\mathrm{col}}^{(s)}(q) \dot{\mathbf{q}}+\mathbf{V}_{\mathrm{col}}^{(v)}(q)\right)
\end{gathered}
$$

Because of the additivity of the collision potentials in Eq. (4), the phase shift for multiple collision $\delta_{\mathrm{col}}$ is a sum of the phase shifts for individual collisions, similar to the case of the Glauber wave function in multiple scattering [4, 13],

$$
\delta_{\mathrm{col}}\left(\kappa x, k x_{d} ; q\right)=\sum_{i}^{N_{\pi}-1} \delta_{\mathrm{col}, i}\left(\kappa x, k x_{d} ; q\right),
$$

where $\delta_{\mathrm{col}, i}\left(\kappa x, k x_{d} ; q\right)$ is obtained from Eqs. (12) and (14) with the potential $v_{\mathrm{col}}\left(q-q_{i}\right)$ in place of the total collision potential $V_{\mathrm{col}}(q)$. The propagation amplitude is therefore

$$
K\left(\kappa x \rightarrow k x_{d}\right)=\int \mathscr{D} q \exp \left\{-i k \cdot\left(x_{d}-x\right)+i \delta_{\mathrm{mf}}\left(\kappa x, k x_{d} ; q\right)+i \delta_{\mathrm{col}}\left(\kappa x, k x_{d} ; q\right)\right\}
$$

If one makes the approximation that the dominant contribution to the path integral comes from the trajectory along the classical path $q_{c}$ for mean-field motion (which need not be a straight line), then the amplitude is approximately

$$
K\left(\kappa x \rightarrow k x_{d}\right) \approx \exp \left\{-i k \cdot\left(x_{d}-x\right)+i \delta_{\mathrm{mf}}\left(\kappa x, k x_{d} ; q_{c}\right)+i \delta_{\mathrm{col}}\left(\kappa x, k x_{d} ; q_{c}\right)\right\} .
$$

For the propagation of an energetic pion, the phase shifts along a straight-line trajectory are just those considered in Ref. [4]. 


\section{TWO-PION CORRELATIONS}

For a pion with momentum $\kappa_{i}$ produced at $x_{i}$ to propagate to momenta $k_{i}$ at the detecting point $x_{d i}$, the amplitude is

$$
\Psi\left(\kappa_{i} x_{i} \rightarrow k_{i} x_{d i}\right)=A\left(\kappa_{i} x_{i}\right) e^{\phi_{0}\left(x_{i}\right)} K\left(\kappa_{i} x_{i} \rightarrow k_{i} x_{d i}\right),
$$

where $A\left(\kappa_{i} x_{i}\right)$ is the production amplitude, and $\phi_{0}\left(x_{i}\right)$ is a random and fluctuating production phase for the chaotic source. The probability amplitude for the production of two identical pions $\left(\kappa_{1}, \kappa_{2}\right)$ at $\left(x_{1}, x_{2}\right)$ to be detected subsequently as $k_{1}$ at $x_{d 1}$ and $k_{2}$ at $x_{d 2}$ is

$$
\frac{1}{\sqrt{2}}\left\{\Psi_{1}\left(\kappa_{1} x_{1} \rightarrow k_{1} x_{d 1}\right) \Psi_{1}\left(\kappa_{2} x_{2} \rightarrow k_{2} x_{d 2}\right)+\left(x_{1} \leftrightarrow x_{2}\right)\right\}
$$

The probability $P\left(k_{1} k_{2}\right)$ for the detection of two pions with momenta $\left(k_{1}, k_{2}\right)$ is the absolute square of the sum of the above amplitudes from all $x_{1}$ and $x_{2}$ source points. Because of the random and fluctuating phase $\phi_{0}\left(x_{i}\right)$ for a chaotic source, the absolute square of the sum of the amplitudes becomes the sum of the absolute squares of the amplitudes [3]. One obtains $P\left(k_{1} k_{2}\right)=P\left(k_{1}\right) P\left(k_{2}\right)\left[1+R\left(k_{1} k_{2}\right)\right]$, where $P\left(k_{i}\right)$ is the probability of detecting a pion of momentum $k_{i}$, and

$$
\begin{aligned}
& P\left(k_{1}\right) P\left(k_{2}\right) R\left(k_{1} k_{2}\right)=\sum_{x_{1} x_{2}} A\left(\kappa_{1} x_{1}\right) A\left(\kappa_{2} x_{2}\right) A\left(\kappa_{1} x_{2}\right) A\left(\kappa_{2} x_{1}\right) \\
& \quad \times K\left(\kappa_{1} x_{1} \rightarrow k_{1} x_{d 1}\right) K\left(\kappa_{2} x_{2} \rightarrow k_{2} x_{d 2}\right) K^{*}\left(\kappa_{1} x_{2} \rightarrow k_{1} x_{d 1}\right) K^{*}\left(\kappa_{2} x_{1} \rightarrow k_{2} x_{d 2}\right) .
\end{aligned}
$$

We can evaluate the product $K\left(\kappa_{1} x_{1} \rightarrow k_{1} x_{d 1}\right) K^{*}\left(\kappa_{2} x_{1} \rightarrow k_{2} x_{d 2}\right)$. It is equal to

$$
\begin{aligned}
& K\left(\kappa_{1} x_{1} \rightarrow\right.\left.k_{1} x_{d 1}\right) K^{*}\left(\kappa_{2} x_{1} \rightarrow\right. \\
& \times \int \mathscr{D} q \int \mathscr{D} q^{\prime} \exp \left\{i \delta_{\mathrm{mf}}\left(\kappa_{1} x_{1}, k_{1} x_{d 1} ; q\right)-i \delta_{\mathrm{mf}}^{*}\left(\kappa_{2} x_{1}, k_{2} x_{d 2} ; q^{\prime}\right)\right. \\
&\left.\quad+i \delta_{\mathrm{col}}\left(\kappa_{1} x_{1}, k_{1} x_{d 1} ; q\right)-i \delta_{\mathrm{col}}^{*}\left(\kappa_{2} x_{1}, k_{2} x_{d 2} ; q^{\prime}\right)\right\} .
\end{aligned}
$$

The real part of the phase difference $\delta_{\text {col }}\left(\kappa_{1} x_{1}, k_{1} x_{d 1} ; q\right)-\delta_{\text {col }}^{*}\left(\kappa_{2} x_{1}, k_{2} x_{d 2} ; q^{\prime}\right)$ is stationary when $q-q^{\prime}=0$ and is random and fluctuating when $q-q^{\prime} \neq 0$. The sum over $\exp \left\{i \delta_{\mathrm{col}}\left(\kappa_{1} x_{1}, k_{1} x_{d 1} ; q\right)-\delta_{\text {col }}^{*}\left(\kappa_{2} x_{1}, k_{2} x_{d 2} ; q^{\prime}\right)\right\}$ is approximately zero when $q-q^{\prime} \neq 0$. Thus, the phase factor $\exp \left\{i \delta_{\mathrm{col}}\left(\kappa_{1} x_{1}, k_{1} x_{d 1} ; q\right)-\delta_{\mathrm{col}}^{*}\left(\kappa_{2} x_{1}, k_{2} x_{d 2} ; q^{\prime}\right)\right\}$ operationally behaves approximately as $\delta\left(q-q^{\prime}\right) \exp \left\{-2 \mathscr{I} m \delta_{\text {col }}\left(\kappa_{1} x_{1}, k_{1} x_{d 1} ; q^{\prime}\right)\right\}$. Upon integrating over $\mathscr{D} q^{\prime}$, we obtain

$$
\begin{aligned}
& K\left(\kappa_{1} x_{1} \rightarrow k_{1} x_{d 1}\right) K^{*}\left(\kappa_{2} x_{1} \rightarrow k_{2} x_{d 2}\right) \approx e^{-i k_{1} \cdot\left(x_{d 1}-x_{1}\right)+i k_{2} \cdot\left(x_{d 2}-x_{2}\right)} \\
& \times \int \mathscr{D} q \exp \left\{i \delta_{\mathrm{mf}}\left(\kappa_{1} x_{1}, k_{1} x_{d 1} ; q\right)-i \delta_{\mathrm{mf}}^{*}\left(\kappa_{2} x_{1}, k_{2} x_{d 2} ; q\right)-2 \mathscr{I} m \delta_{\mathrm{col}}\left(\kappa_{1} x_{1}, k_{1} x_{d 1} ; q\right)\right\} .
\end{aligned}
$$

In the sum over paths in the above integral, the dominant contribution comes from the trajectory $q_{c}$ that minimizes the action difference of the mean field, $\left\{i \delta_{\mathrm{mf}}\left(\kappa_{1} x_{1}, k_{1} x_{d 1} ; q\right)-i \delta_{\mathrm{mf}}^{*}\left(\kappa_{2} x_{1}, k_{2} x_{d 2} ; q\right)\right\}$. We therefore have

$$
\begin{aligned}
& K\left(\kappa_{1} x_{1} \rightarrow k_{1} x_{d 1}\right) K^{*}\left(\kappa_{2} x_{1} \rightarrow k_{2} x_{d 2}\right) \approx \exp \left\{-i k_{1} \cdot\left(x_{d 1}-x_{1}\right)+i k_{2} \cdot\left(x_{d 2}-x_{2}\right)\right. \\
& \left.\quad+i \delta_{\mathrm{mf}}\left(\kappa_{1} x_{1}, k_{1} x_{d 1} ; q_{c}\right)-i \delta_{\mathrm{mf}}^{*}\left(\kappa_{2} x_{1}, k_{2} x_{d 2} ; q_{c}\right)-2 \mathscr{I}_{m} \delta_{\mathrm{col}}\left(\kappa_{1} x_{1}, k_{1} x_{d 1} ; q_{c}\right)\right\} .
\end{aligned}
$$


The other factor in Eq. (20) can be approximated in a similar way. When all the factors are collected, we obtain

where

$$
\begin{gathered}
R\left(k_{1}, k_{2}\right)=\left|\int d^{4} x e^{i\left(k_{1}-k_{2}\right) \cdot x+i \phi_{\mathrm{mf}}\left(k_{1} k_{2}, x ; q_{c}\right)+i \phi_{\mathrm{col}}\left(k_{1} k_{2}, x ; q_{c}\right)} \rho_{\mathrm{eff}}\left(k_{1} k_{2}, x\right)\right|^{2}, \\
\rho_{\mathrm{eff}}\left(k_{1} k_{1}, x\right)=\frac{\sqrt{f\left(\kappa_{1} x\right) f\left(\kappa_{2} x\right)}}{P\left(k_{1}\right) P\left(k_{2}\right)}, \\
\phi_{\mathrm{mf}}\left(k_{1} k_{2}, x ; q_{c}\right)=\int_{x}^{x_{f},\left(\operatorname{path} q_{c}\right)}\left\{\left[p_{1 \mathrm{mf}}(q)-k_{1}\right]-\left[p_{2 \mathrm{mf}}^{*}(q)-k_{2}\right]\right\} \cdot d q,
\end{gathered}
$$

$\phi_{\mathrm{col}}\left(k_{1} k_{2}, x ; q_{c}\right)=\delta_{\mathrm{col}}\left(\kappa_{1} x, k_{1} x_{d 1} ; q_{c}\right)-\delta_{\mathrm{col}}^{*}\left(\kappa_{2} x, k_{2} x_{d 2} ; q_{c}\right) \approx 2 i \mathscr{I}_{m} \delta_{\mathrm{col}}\left(\kappa_{1} x, k_{1} x_{d 1} ; q_{c}\right)$,

and $f(\kappa x)$ is the momentum distribution of the initial chaotic source at $x$ with momentum $\kappa$ that evolves asymptotically to $k$ [4]. The collective flow (mean-field interaction) 'distorts' the initial momentum $\kappa$ into the final detected momentum $k$. The above results from the path-integral method contain those of Ref. [4] as special cases.

The multiple scattering phase $\phi_{\mathrm{col}}\left(k_{1} k_{2}, x ; q_{c}\right)$ can be simplified to be [13]

$$
-2 \mathscr{I}_{m} \delta_{\mathrm{col}}\left(\kappa_{1} x, k_{1} x_{d 1} ; q_{c}\right)=-\int_{x}^{x_{f},\left(\text { path } q_{c}\right)} n_{\pi}\left(q^{\prime}\right) \sigma_{\mathrm{abs}} d q^{\prime}
$$

where $n_{\pi}\left(q^{\prime}\right)$ is the pion density at $\left(q^{\prime}\right)$ and $\sigma_{\text {abs }}$ is the pion absorption cross section.

\section{CONCLUSIONS AND DISCUSSIONS}

In the environment after the phase transition, chemical reactions are essentially completed, and the collisions between pions are predominately elastic. The elastic propagation of a pion can be studied by the Feynman path-integral method. We describe the collective flow of the pion by its dynamical motion in a density-dependent long-range mean-field potential, and its scatterings with other pions by short-range $\pi$ - $\pi$ interactions. We find that HBT correlation measurements lead to an effective source distribution that depends on the initial source distribution at a shifted momentum, with a multiple scattering absorption factor $e^{i \phi_{\mathrm{col}}}$ and a collective flow phase factor $e^{i \phi_{\mathrm{mf}}}$.

The multiple scattering absorption factor depends on the pion absorption cross section. A substantial fraction of the $\pi-\pi$ collisions will go through the $I=1, \rho$ resonance. The width of the $\rho$ meson increases substantially in the medium [9] and the $\rho$ meson mean lifetime in the medium is much shorter than its orbiting time. The scattering of two pions through the intermediary $\rho$ meson is essentially an elastic scattering and does not represent an absorption process in intensity interferometry. Therefore, if one considers the $\pi^{+}-\pi^{+}$correlation, a detected $\pi^{+}$can be absorbed in its propagation through a pion medium only by interacting with a $\pi^{-}$in the $\pi^{+} \pi^{-} \rightarrow \pi^{0} \pi^{0}$ reaction. The cross section $\sigma\left(\pi^{+} \pi^{-} \rightarrow \pi^{0} \pi^{0}\right)$ is equal to $\left(8 \pi / 9 k^{2}\right) \sin ^{2}[\delta(I=0)-\delta(I=2)]$, where $k$ is the magnitude of the pion momentum in the $\pi$ - $\pi$ C.M. system and $\delta(I)$ is the $\pi-\pi$ phase shift for the state of total isospin $I$ [14]. One finds an absorption cross section $\sigma\left(\pi^{+} \pi^{-} \rightarrow \pi^{0} \pi^{0}\right)$ of $\sim 8 \mathrm{mb}$ and a mean absorption path length $\sim 12 \mathrm{fm}$ in a thermalized pion medium at $T=180 \mathrm{MeV}$. The mean absorption path length increases as the temperature decreases. The degree of $\pi^{+}$absorption by the $\pi^{+} \pi^{-} \rightarrow \pi^{0} \pi^{0}$ reaction is small. 
The collective flow leads to a net phase shift $\phi_{\mathrm{mf}}\left(k_{1} k_{2}, x ; q_{c}\right)$ in Eq. (25) that depends on the deviations of the in-medium particle momenta $p_{i \mathrm{mf}}(q)$ from their asymptotic values $k_{i}$. The contributions of the two terms from $p_{1 \mathrm{mf}}$ and $p_{2 \mathrm{mf}}$ in Eq. (25) tend to cancel and give rise only to a small effect, as indicated by similar hydrodynamical calculations where one evaluates $\phi_{\mathrm{mf}}$ by following pion trajectories [6]. Because of the small absorption due to multiple scattering and the small net phase shift due to the collective flow, the effective density measured in HBT measurements is expected to depend on a source distribution close to the initial (chemical freeze-out) source distribution, at a shifted momentum. We expect that as the initial pion source transverse dimension is approximately the spatial dimension of the colliding nuclei, the effective distribution measured by HBT should be nearly independent of the collision energy. We also expect that in the initial source prior to the collective flow, the transverse size in the "out" direction should be approximately the same as that in the "side" direction. Hence, $R_{\text {out }} / R_{\text {side }}$ should be approximately close to 1 . These expectations are consistent with the gross features of HBT transverse radii in high-energy heavy-ion collisions. It will therefore be of great interest to carry out numerical calculations of the evolution of the produced hadron matter to study the above effects.

\section{ACKNOWLEDGMENTS}

The author thanks Profs. H. Crater, R. J. Glauber, Chi-Sing Lam, and Weining Zhang for helpful discussions. This research was supported in part by the Division of Nuclear Physics, U.S. Department of Energy, under Contract No. DE-AC05-00OR22725, managed by UT-Battelle, LLC and by the National Science Foundation under contract NSFPhy-0244786 at the University of Tennessee.

\section{REFERENCES}

1. R. Hanbury-Brown and R. Q. Twiss, Phil. Mag. 45, 663-682 (1954).

2. R. J. Glauber, Phys. Rev. Lett. 10, 84-86 (1963); R. J. Glauber, Phys. Rev. 130, 2529-2539 (1963).

3. For a general review of the Hanbury-Brown-Twiss intensity interferometry, see Chapter 17 of C. Y. Wong, Introduction to High-Energy Heavy-Ion Collisions, World Scientific Pub. Company, 1994.

4. C. Y. Wong, J. Phys. G29, 2151-2168 (2003).

5. C. Y. Wong, J. Phys. G30, S1053-S1058 (2004).

6. W. N. Zhang, M. J. Efaaf, C. Y. Wong, and M. Khaliliasr, Chin. Phys. Lett. 21, 1918-1921 (2004).

7. J. Kapusta and Y. Li, J. Phys. G30, S1069-S1072 (2004).

8. J. G. Cramer, G. A. Miller, J. M. S. Wu, and Jin-Hee Yoon, Phys. Rev. Lett. 94, 102302 (2005); J. G. Miller and G. A. Cramer, nucl-th/0507004.

9. M. Urban, M. Buballa, and J. Wambach, Phys. Rev. Lett. 88, 042002 (2002).

10. C. Y. Wong, E. S. Swanson, and T. Barnes, Phys. Rev. C65, 014903 (2002).

11. C. Y. Wong, J. Maruhn, and T. Welton, Nucl. Phys. A253, 469-489 (1975); C. Y. Wong and H. H. K. Tang, Phys. Rev. Lett. 40, 1070-1073 (1978); C. Y. Wong and H. H. K. Tang, Phys. Rev. 20, 1419-1452 (1979); C. Y. Wong, Phys. Rev. 25, 1460-1475 (1982).

12. For a recent review on the path-integral method, see H. Kleinert, Path Integrals in Quantum Mechanics, Statistics, Polymer Physics, and Financial Markets, World Scientific Pub. Company, Singapore 2004, Fourth Extended Edition.

13. C. Y. Wong and R. J. Glauber, to be published.

14. B. R. Martin, D. Morgan, and G. Shaw, Pion-Pion Interaction in Particle Physics, Academic Press, 1976, p.101. 